\title{
The Research on Time Synchronization Protocol of Lightweight for Wireless Sensor Network
}

\author{
Peng $\mathrm{Lu}^{1+}, \mathrm{Xiao} \mathrm{Cong}^{2}$ and $\mathrm{Zhe} \mathrm{Cao}^{3}$ \\ ${ }^{1}$ Department of Media Technology and Communication, Northeast Electric Power University, Jilin, Jilin, \\ China \\ ${ }^{2}$ College of Science, Northeast Electric Power University, Jilin, Jilin, China \\ ${ }^{3}$ Ideal Research Institute of Information Technology, Northeast Normal University, Changchun, Jilin, China
}

\begin{abstract}
Time synchronization technology is a challenging area of research in wireless sensor network. The diversity of wireless sensor network leads to the diversity of demands on the time synchronization mechanism; therefore, it is impossible to use one kind of time synchronization mechanism to satisfy all application requirements. Due to its own characteristics, wireless sensor network needs special requirements in synchronization precision, synchronization scope, and energy consumption etc. This paper presents a lightweight and active time synchronization protocol for wireless sensor network, being called LATS for short. It ensures that the synchronization accuracy in practical applications meets the circumstances while reducing the energy costs as far as possible. Finally, the performance of this protocol is proved by experiment.
\end{abstract}

Keywords: WSN, Time Synchronization, Protocol, Active, Lightweight

\section{Introduction}

The wireless sensor network(WSN) consists of a large number of micro sensor nodes using wireless adhoc mode [1], [2], it is known as one of the most important technology of the 21 st century and widely used [3], such as utilities, environmental monitoring, patient health monitoring, smart home, etc.

As with all distributed systems, an important problem of WSNs is time synchronization of all nodes. Many key technologies require the clock of sensor nodes are synchronized in the WSNs, such as data fusion, co-processing, low-power design, TDMA, synchronization, and other key technology sleep cycle all sensor nodes in the network requires synchronized clocks.

The time synchronization requirements of WSN are very different with other distributed systems. Large size and node density of the network, thus requiring the synchronization protocol should be able to adapt to changes in the number of nodes, and the power of sensor nodes is very limited, so the efficient use of energy is also to be considered one of the main problem [4]. Currently proposed the famous time synchronization technology for WSNs is that the reference clock source for time synchronization to send messages to other nodes synchronized clocks via active way, such as RBS, TPSN, and DMTS. In fact, with respect to the reference clock source for those nodes that have very accurate clock do not require time synchronization. Therefore, the above time synchronization mechanism is bound to waste energy of nodes when sending receiving time synchronization messages. To solve this problem, we propose a new protocol which called a lightweight and active time synchronization protocol (LATS) in this paper, it fully into account the characteristics of WSNs and less overhead, but also has better robustness and self-organization.

\section{Time Synchronization Protocol}

\footnotetext{
+ Corresponding author. Tel.: + 86-15981162821.

E-mail address:lup595@nenu.edu.cn.
} 
The design of time synchronization protocol should consider many specific applications in WSNs, such as energy, accuracy, robustness and so on. In this section, we first detailed analysis of the various constraints of time synchronization protocol, then design a lightweight active time synchronization protocol and introduces the principle and implementation.

\subsection{The Time Synchronization Protocol of WSNs}

Widely used time synchronization mechanism are Global Positioning System (GPS) [5] [6] [7] and Network Time Protocol (NTP) [8] [9], but the main purpose of the two mechanisms is how to get highprecision time synchronization, and the algorithm complicated and expensive. Due to the characteristics of WSNs itself which is required to reduce the synchronization algorithm complexity under certain synchronization accuracy, and to minimize the node energy consumption. Therefore, the traditional time synchronization technology is not applicable to WSNs [10]. Therefore, it need to trade-offs between accuracy and energy efficiency depending on the applications.

\subsection{The Time Synchronization Protocol of WSNs}

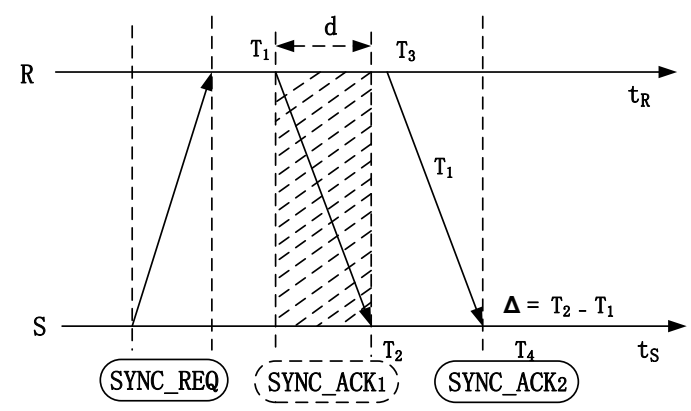

Fig. 1: Peer to peer synchronization process

As shown in Fig.1, node $S$ is the node to be synchronized and $t_{S}$ is time of $S$; node $R$ is the clock reference source node and $t_{R}$ is time of $R$, the synchronization process of LATS is as follows:

Step 1: To be synchronized node $\mathrm{S}$ (sub-node) sends a time synchronization re-quest message SYNC_REQ to the clock reference node R (parent-node), and start time synchronization.

Step 2: After node $\mathrm{R}$ receives the time synchronization request message from $\mathrm{S}$, it is immediately send a start time synchronization message SYNC_ACK 1 to $\mathrm{S}$, and the body of SYNC_ACK S $_{1}$ without any contents. At the same time, the node $\mathrm{R}$ recording time $\mathrm{T}_{1}$ to send the message $\mathrm{SYNC}_{-} \mathrm{ACK}_{1}$ in the MAC layer.

Step 3: After send SYNC_ACK ${ }_{1}$, then the node $\mathrm{R}$ packed time $\mathrm{T}_{1}$ previously collected into synchronous message SYNC_ACK 2 , then send SYNC_ACK 2 to node S.

Step 4: When node $\mathrm{S}$ receive synchronization message $\mathrm{SYNC}_{-} \mathrm{ACK}_{1}$, and record the time $\mathrm{T}_{2}$ was received message in MAC layer.

Step 5: At time $\mathrm{T}_{4}$, after node $\mathrm{S}$ receive synchronous message $\mathrm{SYNC}_{-} \mathrm{ACK}_{2}$, then unpacked from the message body and get the time $\mathrm{T}_{1}$ and perform the following calculation: $\Delta=\mathrm{T}_{2}-\mathrm{T}_{1}$.

Step 6: So get the time difference $\Delta$ between the node $\mathrm{S}$ waiting for synchronization and reference clock node $\mathrm{R}$, then node $\mathrm{S}$ adjust its own local clock according to $\Delta$ and synchronized with the clock reference node $\mathrm{R}$.

The LATS protocol full account of the transmission delay to critical path which synchronization messages from the sending node to the receiving node on.

Firstly, rather than the other time synchronization mechanisms which passive to accept time synchronization from reference source, the nodes which need to be synchronized time initiative sends a synchronization message to the clock reference node in LATS protocol, this can avoid the situation which the node has very accurate but have to passively accept the time synchronization, so reducing the number of synchronization messages and saves power.

Secondly, the clock reference node immediately sends a synchronization message to the node which needs to be synchronized after receiving the message, and this message body does not contain any 
information, just tell the node that start time synchronization. Meanwhile, the clock reference node also recording own present time in MAC layer. This will eliminate the effects of send time delay and access time delay to the synchronization accuracy.

Thirdly, since the distance between nodes mostly within 100 meters, the transmission time delay of message on the link nanosecond level mostly, therefore, the transmission time delay is negligible.

Finally, as the father node immediately sent a second time synchronization message after the first synchronization message sent over, and deviation caused by crystal frequency negligible, so $T_{3}-T_{1}=T_{4}-T_{2}$. When treatment the second time synchronization message received from clock reference node, the errors may occur of time accuracy of node to be synchronized due to the deviation of the crystal. However, for lightweight applications this error can be ignored for practical application will not be affected.

\subsection{The Entire Network Time Synchronization Based on Layer Structure}

To establish hierarchical tree topology of time synchronization protocol which is established in the early stages of network deployment. First, the node that need to join the network sends network application, such as $\mathrm{N}_{2}$ in the following Fig. 2; Next, and nodes in network that agreed the application reply node $\mathrm{N}_{2}$. In fact, it may include Base Station, $\mathrm{N}_{1}, \mathrm{~N}_{3}$ in the figure; Next, accordance with the requirements, $\mathrm{N}_{2}$ select the optimal node as its parent node in reply of all nodes. Specifically, it uses of simulated annealing algorithm [11] [12] to choose the parent node. The algorithm has characteristics such as high efficiency, robustness, versatility, flexibility and so on [13] [14]. The selection process of parent node by simulated annealing algorithm. In this case, the parent node is Base Station. Then, the node $\mathrm{N}_{2}$ with the parent node Base Station for time synchronization. The time synchronization of first layer nodes with the Base Station, and the time synchronizations of second layer nodes with the first layer nodes, and so on. This process continues until all nodes in network have the same time with Base Station.

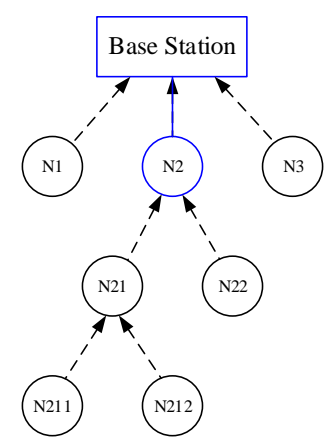

Fig. 2: The entire network time synchronization

In the WSNs that the maximum number of hops is $n$ of node, the total error of time synchronization is $n$ times of the single-hop synchronous error in the worst case. As single-hop error may also be negative as positive in multi-hop network, therefore, the sum of the multi-hop synchronization errors can offset some of the single-hop error. The LATS protocol conducted a compromise between implementation complexity, energy-efficient and synchronization accuracy, and can be used in WSNs which requirement the time synchronization is not very high.

\section{Analysis of Results}

For the proposed LATS protocol, through experiments conducted detailed testing. Set reference_node is clock reference node, and request_node is synchronized node. The accumulated values of reference_node and request_node according to the local oscillator before the time synchronization, so the time curve is monotonically increasing. Then, due to the different initial time, the starting point of the two curves (i.e., initial phase) is different, in addition, two curves are not completely linear due to clock drift rate. In physical time at the scale of 18, request_node sends a request for time synchronization and reference_node respond to the request. After synchronization is complete, request_node time synchronization to the reference_node, and therefore have time curve after coincidence, so the two time curves coincide together. The curve as shown in Fig.3 that the LATS protocol is feasible and can achieve the purpose of time synchronization. 


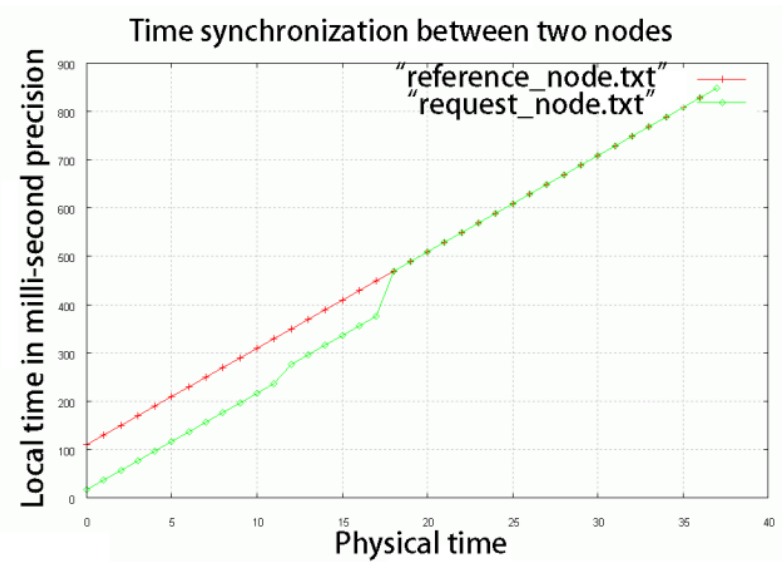

Fig. 3: Time synchronization between two nodes

\subsection{Single-hop Synchronization Error analysis}

Since the clock reference node and synchronization node still in accordance with their crystal cumulative time value respectively, it still will be deviations between the two nodes over the physical time. The time deviation curve between two nodes as shown in Fig. 4 after synchronization as physical time goes on.



Fig. 4: The time deviation between two nodes after synchronization

As shown in above figure, the deviation between two nodes is 17.04 milliseconds after 12 scale (each scale is 5 minutes), and the deviation is 29.01 milliseconds after 2 hours ( 24 scale). Therefore, in order to maintain a relatively accurate of time and the node requires periodic time synchronization which the synchronous cycle needs to be designed according to actual application requirements.

\subsection{Multi-hop Synchronization Error analysis}

In wireless sensor networks, multi-hop error that must be considered. So build a tree topology of 8 layers in testing.

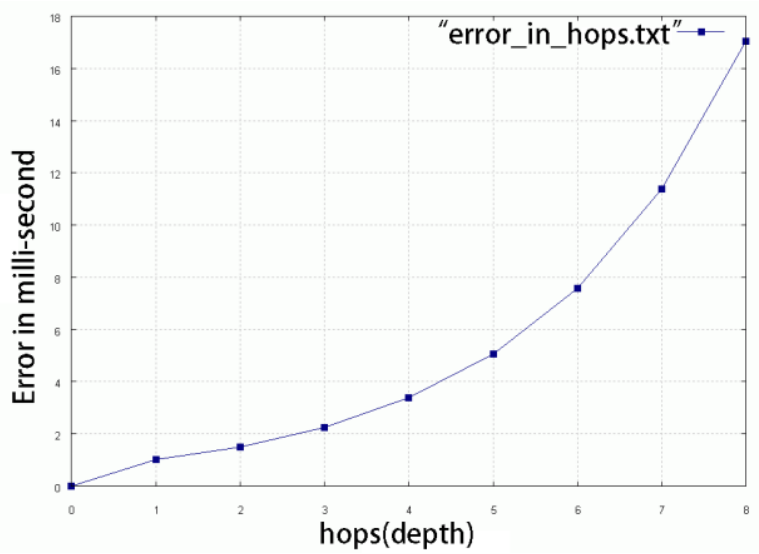

Fig. 5: The time error of every layer's respect to clock reference source after synchronization 
As shown in Fig. 5, the synchronization error of nodes in every layers increases with the number of layers after the completion of the entire network time synchronization. In the experiment, the measured error between 8 th layer and the 1st layer reference node is 17.06 milliseconds.

\section{Conclusion}

The proposed lightweight active time synchronization protocol first to build a hierarchy tree within the network, then start from the Base Station, a node between the adjacent layers pairwise time synchronization mechanism for time synchronization, all nodes between adjacent layers take pairwise mechanism for time synchronization. Finally, to achieve a time synchronization of the whole network. After testing over the corresponding hardware and software systems and complete the time synchronization. The LATS protocol with less overhead to achieve the accuracy required of the entire network time synchronization to prove that the protocol is feasible, and laid a good foundation for the practical application. Because of this work, the time synchronization mechanism will be further studied. Such as portability of protocol and need to consider the new technology of to obtain the time stamp from MAC layer that reduces the number of messages interactively and ultimately reduce the energy consumption of the synchronization algorithm.

\section{References}

[1] Fengyuan, R., Haining, H., Chuang, L. Wireless sensor network. Journal of Software, 14 (7), pp. 1282-1291, 2003.

[2] Galina S. Panayotova, Georgi P. Dimitrov, and Dimitar A. Dimitrov .Wireless Sensors for Analysis Transport Systems. Journal of Communications, 13(1), pp. 40-44, 2018.

[3] Hsieh, T. T. Using Sensor Networks for Highway and Traffic Applications. Potentials IEEE, 23(2), pp. 13-16, 2004.

[4] Jana, V. G., Jan, R. Lightweight Time Synchronization for Sensor Networks. ACM, pp. 11-19, 2003.

[5] Hofmann, W., Lichtenegger, H., Collins, J. Global Positioning System: Theory and Practice. SpingerWien, New York, 1997.

[6] Fatiha Mekelleche and Hafid Haffaf. Classification and Comparison of Range-Based Localization Techniques in Wireless Sensor Networks. Journal of Communications, 12(4), pp. 221-227, 2017.

[7] Duc Phuc Nguyen, Thi Hong Tran, and Yasuhiko Nakashima. A Multi-Mode Error-Correction Solution Based on Split-Concatenation for Wireless Sensor Nodes. Journal of Communications, 12(2), pp. 130-136, 2017.

[8] Yanwei, H., Zheng, Q., Zhongzhi, Z. Intrusion detection algorithm based on simulated annealing and K-mean Clustering. Computer Science, 37(6), pp.122-124, 2010.

[9] Wei Peng, Dongyan Chen, Wenhui Sun, and Guiqing Zhang. Distributed Adaptive Channel Allocation in MultiRadio Wireless Sensor Networks. Journal of Communications, 11(11), pp. 984-991, 2016.

[10] MILLSDL. Internet Time Synchronization: The Network Time Protocol. IEEE Transaction on Communications, 39 (10), pp.1482-1493, 1991.

[11] Sivrikaya, F., Yener, B. Time synchronization in sensor networks: a survey. IEEE Network, 18(4), pp.45-50, 2004.

[12]Defu, Z., Yu, P., Wenxing, Z., Huowang, C. A Hybrid Simulated Annealing Algorithm for the Three-Dimensional Packing Problem. Chinese journal of computer, 32(11), pp.2147-2156, 2009.

[13] Wei, S., Qiang, L.: Business Process Mining Based on Simulated Annealing. Acta Electronica Sinica, 36(4A), pp.135-139, 2008.

[14]Hervé Gokou Diédié, Michel Babri, and Boko Aka. Efficient Cluster-Based Self-Organization Scheme for Connectivity Control in Wireless Sensor Networks. Journal of Communications, 11(7), pp. 632-643, 2016. 